\title{
Endoscopic Ultrasound Biopsy
}

National Cancer Institute

\section{Source}

National Cancer Institute. Endoscopic Ultrasound Biopsy. NCI Thesaurus. Code C112516.

The use of ultrasound imaging for real-time guidance of an endoscope for a biopsy

procedure. 